\title{
Tuning optical properties of Ge nanocrystals by Si shell
}

\author{
M.O. Nestoklon, ${ }^{1}$ A.N. Poddubny,${ }^{1}$ P. Voisin,${ }^{2}$ and K. Dohnalova ${ }^{3}$ \\ ${ }^{1}$ Ioffe Institute, 194021 St. Petersburg, Russia \\ ${ }^{2}$ Laboratoire de Photonique et Nanostructures, CNRS \\ and Université Paris-Saclay, Route de Nozay, 91460 Marcoussis, France \\ ${ }^{3}$ Van der Waals-Zeeman Institute, University of Amsterdam, \\ Science Park 904, NL-1098 XH Amsterdam, The Netherlands
}

\begin{abstract}
We present a theoretical study of Ge-core/Si-shell nanocrystals in a wide bandgap matrix and compare the results with experimental data obtained from the samples prepared by co-sputtering. The empirical tight-binding technique allows us to account for the electronic structure under strain on the atomistic level. We find that a Si shell as thick as 1 monolayer is enough to reduce the radiative recombination rate as a result of valley $L-X$ cross-over. Thin $\mathrm{Si}$ shell leads to a dramatic reduction of the optical bandgap from visible to near-infrared range, which is promising for photovoltaics and photodetector applications.

Our detailed analysis of the structure of the confined electron and hole states in real and reciprocal spaces indicates that the type-II heterostructure is not yet achieved for Si shells with the thickness below $0.8 \mathrm{~nm}$, despite some earlier theoretical predictions. The energy levels of holes are affected by the Si shell stronger than the electron states, even though holes are completely confined to the Ge core. This occurs probably due to a strong influence of strain on the band offsets.
\end{abstract}

\section{INTRODUCTION}

Ge nanostructures are of great interest for light emitter/detector applications in optoelectronics, photonics and photovoltaics. ${ }^{1}$ The indirect bandgap $k$-vector selection rules are lifted in nanostructures by quantum confinement, promising greatly enhanced radiative rate and size-tunable bandgap. ${ }^{2-6}$ Unfortunately, Ge nanocrystals ( $\mathrm{NCs}$ ) naturally oxidize upon exposure to air, leading to formation of O-related centers that act either as radiative centers with fast decaying blue-green emission ${ }^{2,7-9}$ or as defect traps dramatically reducing emission (see e.g. remarks in Ref. 2). Despite these difficulties, Ge nanostructures remain of great interest, showing attractive properties like highly efficient space separated multiple exciton generation (MEG). ${ }^{10}$ Moreover, for integrated optoelectronics and photonics development, Ge remains to be cheaper and more CMOS compatible counterpart to Si than III-V and II-VI semiconductors.

A possible route to further advanced material properties engineering is alloying and straining of $\mathrm{Ge}$ by $\mathrm{Si}$ by developing various $\mathrm{Si} / \mathrm{Ge}$ nano-heterostructures. ${ }^{11,12}$ Alloying $\mathrm{Ge}$ with $\mathrm{Si}$ allows one to tune bandstructure and bandgap into almost optimal range for photovoltaics employing carrier multiplication. ${ }^{13-15}$ Tensile straining of Ge can lead to modified band structure towards direct bandgap, ${ }^{1,16-18}$ which is more pronounced compared to $\mathrm{Si}$, due to a small energy difference between the direct $\Gamma-\Gamma$ and the indirect $(\Gamma-L)$ transitions. Possible routes towards permanent straining are core-shell type heterostructures, such as nanowires or nanocrystals. Si and Ge form type-II heterostructures and it is natural to expect that in the $\mathrm{Si} / \mathrm{Ge}$-based nanostructures the holes are localized in the Ge region and electrons in the Si region. However, there is a lattice mismatch of $4 \%$ between the $\mathrm{Si}$ and $\mathrm{Ge}$, and consequently, band alignment is modified by the strain. Ge NCs capped with thin Si shell are also promising because $\mathrm{Si}$ acts as a protective coating, eliminating formation of the $\mathrm{GeO}$ oxide layer, ${ }^{19}$ source of emissive defect centers ${ }^{20,21}$ and/or emission quenching. ${ }^{22}$ Type-II heterostructures are in general interesting for photodetectors and memory applications ${ }^{19}$ due to lowered rates of optical transitions, as spatially separated electron and hole recombine less efficiently. Also, photon absorption process is typically controlled by the core-core or shell-shell transition, that is energetically shifted from the core-shell emission. Hence, such system also provides transparency for emission due to the lack of reabsorption, interesting for amplifiers and lasers. Core-shell scheme can also lead to reduced blinking ${ }^{23}$ and single exciton population inversion. ${ }^{24}$

Quite recently, unusually bright and fast decaying ( $\sim 1 \mathrm{~ns})$ photoluminescence (PL) has been reported from co-sputtered $\mathrm{Ge}$ and Si-rich $\mathrm{SiO}_{2} \cdot{ }^{25}$ Three-component PL has been observed, strongly suggesting formation of type-II core-shell heterostructure, however, this suggestion was not confirmed. The properties of such coreshell nanocrystals were discussed in the literature mostly based on $k \cdot p$ approach that lacks sensitivity to valleydependent phenomena, crucial in understanding the electronic structure of the system. Also, not much emphasis has been put on complex strain in the realistic nanocrystals which could further obscure the details of their electronic structure.

The ways to prepare well-defined core-shell $\mathrm{Si} / \mathrm{Ge}$ or $\mathrm{Ge} / \mathrm{Si} \mathrm{NC}$ systems are relatively scarce. In past, various techniques were used, such as mechanical alloying by ball milling, ${ }^{26}$ wet chemical etching ${ }^{27}$ and molecular beam epitaxy, ${ }^{28-30}$ wet chemical synthesis ${ }^{31,32}$, synthesis from gas phase (silane and germane) $)^{33-36}$ and cosputtering. ${ }^{37-39}$ The segregation of $\mathrm{Ge}$ and $\mathrm{Si}$ is often the case for Ge-rich SiGe alloys, even though miscibility of $\mathrm{Ge}$ and $\mathrm{Si}$ is expected for whole compositional range. It has been shown both theoretically and experimentally 
that Si-capped Ge NCs have formation energies similar to those of Ge NCs with the same number of atoms, while Ge-capped Si NCs are less stable than Si NCs. ${ }^{40}$ For samples with high Ge content prepared by radio frequency (RF) magnetron co-sputtering and annealed at high temperatures, composite NCs consisting of a Ge NC core and amorphous SiGe shell have been reported from the Raman analysis. ${ }^{39,41}$ Currently, the best evidence for formation of Ge-core/Si-shell systems has been provided by M. Buljan using co-sputtering technique and X-ray analysis. $^{38}$

Available Density Functional Theory (DFT) calculations of small core-shell $\mathrm{Si} / \mathrm{Ge}$ and $\mathrm{Ge} / \mathrm{Si} \mathrm{NC}$ systems by Ramos et al. ${ }^{40}$ and Oliveira et al. ${ }^{42}$ predict type-II confinement with holes in Ge and electrons in Si. Si/Ge core-shell system is shown to offer high oscillator strength and long decay time, but large shift of absorption threshold with varying Ge shell thickness. Ge/Si core-shell, on the other hand shows long lifetimes with lower absorption shifts, making this material more suitable for e.g. photodetectors. For larger $\mathrm{Si} / \mathrm{Ge}$ and $\mathrm{Ge} / \mathrm{Si}$ coreshell NC systems, tight binding calculations are available by Neupane et al. ${ }^{43,44}$, however, with limited scope: study of carriers in $\mathrm{Ge} / \mathrm{Si}$ core-shell system with thick fixed shell thickness of $5 \mathrm{~nm}^{43}$ and in large Ge/Si domeshaped nanostructures with the base diameters in the range $5 \div 45 \mathrm{~nm} .{ }^{44}$ In our work we address the missing category of larger Ge NC core with thin Si shell: system that can be relatively simply prepared by chemical synthesis $^{31}$ or sputtering. ${ }^{38}$ New tools have been developed to trace real- and $k$-space origin for each quantumconfined state. Using these tools we show that Ge optical properties can be extensively tuned by adding relatively thin Si shell.

\section{TIGHT-BINDING MODEL}

The structure under consideration is schematically shown in Fig. 1(a). It consists of a Ge nanocrystalline core with the diameter $D_{\text {core }}$ (red atoms), and crystalline $\mathrm{Si}$ shell with the diameter $D_{\text {shell }}$ (green atoms) embedded in the amorphous $\mathrm{SiO}_{2}$ matrix (gray atoms). The atomic coordinates between $\mathrm{Si}$ and $\mathrm{SiO}_{2}$ were relaxed according to the standard valence force field (VFF) theory $^{45}$ in order to minimize the strain energy. This approach is reasonable for covalent semiconductors and provides atomistic strain with relatively high precision. ${ }^{46}$ We model $\mathrm{SiO}_{2}$ as a virtual crystal with zincblende lattice, with bandstructure near band gap similar to $\alpha$ quartz. In order to describe nominally unstrained coreshell $\mathrm{Si} / \mathrm{Ge} \mathrm{NC}$, we set the VFF parameters to mimic soft material. The electronic spectrum has been obtained using the standard $s p^{3} d^{5} s^{*}$ empirical tight-binding (TB) technique ${ }^{47}$ and parametrization. ${ }^{48}$ Since there is no universally accepted TB parametrization of $\alpha-\mathrm{SiO}_{2}$ in literature, ${ }^{49,50}$ we have considered $\mathrm{SiO}_{2}$ phenomenologically, with TB parameters manually chosen to reproduce (a)

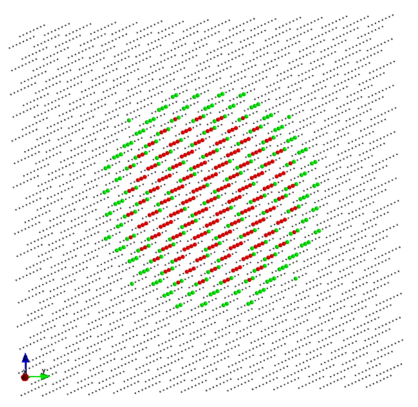

(b)

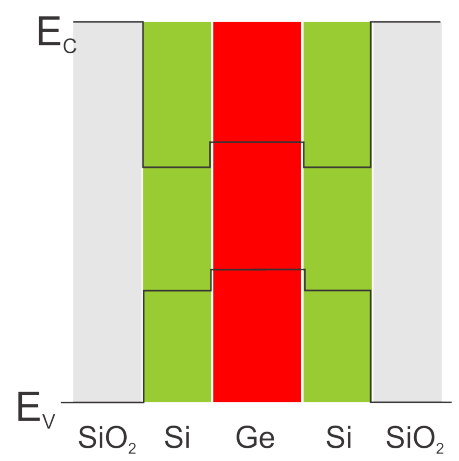

FIG. 1. (Color online) Schematic sketch of studied $\mathrm{Ge} / \mathrm{Si}$ core/shell NCs system. (a) 3D plot of atomic arrangement in one of the real structures calculated. Ge atoms are shown by red dots, $\mathrm{Si}$ atoms are green and atoms which simulate $\mathrm{SiO}_{2}$ matrix are gray. (b) Sketch of nanocrystal band structure. (a)

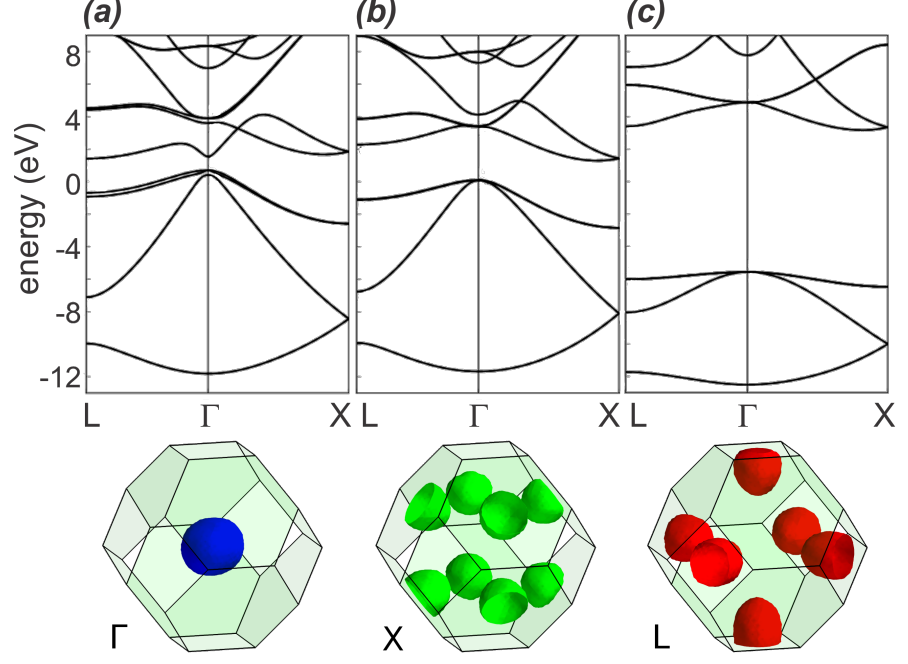

FIG. 2. (Color online) Tight-binding band structure of bulk (a) Ge, (b) Si and (c) $\mathrm{SiO}_{2}$. (d) Brillouin zone with isosurfaces of $k$-space LDOS (see below) for typical electron states confined in $\Gamma, X$ and $L$ valleys for a real NC.

the experimental band gap and offsets. ${ }^{4}$ The valence and conduction band offsets between $\mathrm{Si}, \mathrm{Ge}$ and $\mathrm{SiO}_{2}$ lead to the type-II heterostructure system, the schematic band diagram is depicted in Fig. 1(b). The tight-binding parameters used are given in supplementary material. The corresponding TB band structures of bulk Ge, Si and $\mathrm{SiO}_{2}$ are shown in Fig. 2(a-c), respectively. The bulk $\mathrm{Si}$ and Ge have quite different band structures with the conduction band minima located in the $X$ and $L$ valleys, respectively. The valence band extrema, however, are in the $\Gamma$ point for all considered materials.

NC have discrete set of eigenstates with energy $E_{i}$ and wavefunction $\psi_{i}$. The TB wavefunction of $i$-th state (we 

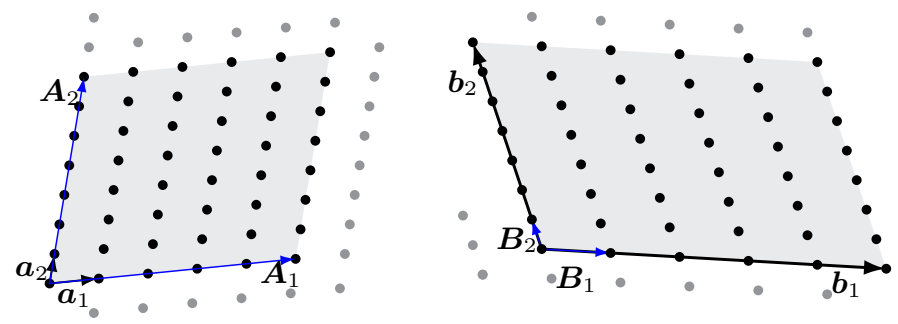

FIG. 3. Illustration of atom real space $\left(\boldsymbol{a}_{i}, \boldsymbol{A}_{i}\right)$ and reciprocal $\left(\boldsymbol{b}_{i}, \boldsymbol{B}_{i}\right)$ space basis vectors of lattice $\left(\boldsymbol{a}_{i}, \boldsymbol{b}_{i}\right)$ and supercell $\left(\boldsymbol{A}_{i}, \boldsymbol{B}_{i}\right)$.

will omit it below when this does lead to confusion)

$$
\psi_{i}=\sum_{n, \alpha} C_{n \alpha}^{(i)} \Psi_{\alpha}\left(\boldsymbol{r}-\boldsymbol{r}_{n}\right)
$$

is determined by a set of complex coefficients $C_{n \alpha}^{(i)}$ defined on a lattice $\boldsymbol{r}_{n}$ in $3 \mathrm{D}$ space. The index $\alpha$ labels the orbital and spin degrees of freedom of the atomic-like wavefunctions $\Psi_{\alpha}(\boldsymbol{r})$. Tight-binding wave functions are hard to analyze without a postprocessing that extracts physically relevant quantities. An obvious example is the real space distribution of electron density: local density of states (LDOS) which we define as

$$
n^{i}(\boldsymbol{r})=\sum_{n, \alpha}\left|C_{n, \alpha}^{(i)}\right|^{2} \frac{e^{-\left(\boldsymbol{r}-\boldsymbol{r}_{n}\right)^{2} / a^{2}}}{(\sqrt{\pi} a)^{3}}
$$

where $C_{n \alpha}$ are the tight-binding coefficients at $n$-th atom, $a$ is Gaussian broadening which may be chosen to be of the order of interatomic distance and $\boldsymbol{r}_{n}$ is the position of $n$-th atom. Equation (2) for LDOS allows one to trace the real space distribution of the electron density for the particular state. However, LDOS only is not enough to visualize all the features of electron states. In order to trace the valley structure of the states, below we define k-space LDOS. For this, we perform a Fourier transform of the TB wavefunction of confined carriers as explained below. For a given lattice $\boldsymbol{r}_{n}$ one can formally define a lattice in the reciprocal space. ${ }^{51}$ For the finite system we consider a large supercell and apply periodic boundary conditions. The supercell is defined as a parallelogram cut from ideal zincblende lattice by vectors $\mathbf{A}_{i}, i=1 . .3$. This parallelogram defines a lattice in the reciprocal space with the basis vector

$$
\mathbf{B}_{1}=2 \pi \frac{\mathbf{A}_{2} \times \mathbf{A}_{3}}{\mathbf{A}_{\mathbf{1}} \cdot\left[\mathbf{A}_{2} \times \mathbf{A}_{3}\right]}
$$

and $\mathbf{B}_{2}, \mathbf{B}_{3}$ are obtained by the cyclic permutations.

Positions of cations in the ideal lattice are defined as

$$
\mathbf{r}_{n_{1} n_{2} n_{3}}=\mathbf{a}_{1} n_{1}+\mathbf{a}_{2} n_{2}+\mathbf{a}_{3} n_{3}
$$

where $\mathbf{a}_{i}$ are the standard basis vectors of face-centered cubic lattice and anions are shifted by the vector $a_{0} / 4(1,1,1)$. The vectors $\mathbf{a}_{i}$ correspond to the reciprocal space vectors

$$
\mathbf{b}_{1}=2 \pi \frac{\mathbf{a}_{2} \times \mathbf{a}_{3}}{\mathbf{a}_{1} \cdot\left[\mathbf{a}_{2} \times \mathbf{a}_{3}\right]}, \text { and c.p. }
$$

The vectors $\mathbf{b}$ form the basis of another lattice in the reciprocal space associated with the crystal lattice. The first Brillouin zone of the lattice is defined by the supercell vectors $\boldsymbol{B}_{i}$ by considering vectors $\mathbf{k}_{n_{1}, n_{2}, n_{3}}=$ $\mathbf{B}_{1} n_{1}+\mathbf{B}_{2} n_{2}+\mathbf{B}_{3} n_{3}$ equal if and only if they are differ by a linear combination of $\mathbf{b}_{i}$. We will call this set of vectors "k-space mesh"

$$
\begin{array}{r}
\mathcal{K}_{\mathrm{BZ}}=\left\{\mathbf{k}_{n_{1}, n_{2}, n_{3}} \mid \mathbf{k}_{n_{1}, n_{2}, n_{3}}-\mathbf{k}_{n_{1}^{\prime}, n_{2}^{\prime}, n_{3}^{\prime}} \neq m_{1} \mathbf{b}_{\mathbf{1}}\right. \\
\left.+m_{2} \mathbf{b}_{\mathbf{2}}+m_{3} \mathbf{b}_{\mathbf{3}}\right\}
\end{array}
$$

Simple linear algebra shows that the number of vectors $\mathbf{k}_{n_{1}, n_{2}, n_{3}}$ in the first Brillouin zone is exactly equal to the number of prime cells in a supercell bounded by the vectors $\mathbf{A}_{i}$. Definition of the vectors $\mathbf{a}_{i}, \mathbf{A}_{i}, \mathbf{b}_{i}$ and $\mathbf{B}_{i}$ is illustrated in Fig. 3. With these definitions we introduce a discrete Fourier transform by

$$
C_{l \tau \alpha}^{\mathbf{k}}=\sum_{n \in \text { sublattice } \tau} C_{n \alpha} e^{i \mathbf{r}_{n} \mathbf{k}_{l}}, \quad \mathbf{k}_{l} \in \mathcal{K}_{\mathrm{BZ}}
$$

that gives us the same number of coefficients as we had in the TB wavefunction in real space. The sum in Eq. (7) runs through atoms in two sublattices separately. This formal definition satisfies the obvious properties one could expect from a function in reciprocal space: the norm is conserved, the orthogonality of the states is preserved. If one considers the state with well-defined wave vector $\mathbf{k}_{0}$, the corresponding Fourier transform $C_{l \tau \alpha}^{\mathbf{k}}$ has a sharp maximum for $\mathbf{k}_{l} \sim \mathbf{k}_{0}$. This allows us to attribute the confined states to particular valleys.

Similar to LDOS in real space Eq. (2) we may define LDOS in reciprocal space, which we will call k-space LDOS or kLDOS below, as

$$
n^{i}(\boldsymbol{k})=\sum_{l \tau \alpha}\left|C_{l \tau \alpha}^{\mathbf{k}(i)}\right|^{2} \frac{e^{-\left(\boldsymbol{k}-\boldsymbol{k}_{l}\right)^{2} / q^{2}}}{(\sqrt{\pi} q)^{3}},
$$

where the $k$-space Gaussian smoothing parameter $q$ is conveniently chosen of the order of $\left|\mathbf{B}_{i}\right|$. To illustrate this procedure, Fig. 2(d) presents the isodensity surface plots of $k$-space LDOS calculated using Eq. (8) for few particular states which show definite $\Gamma, L$ and $X$ valley character for a realistic calculations of NCs, see below. It can be seen that the kLDOS indeed clearly shows the valley structure.

Importantly, this approach can be formally used for strained structures as well, although care is needed. When the strain is applied, atomic positions start to deviate from ideal crystal lattice and a proper generalization of the above scheme must be used. The approach outlined above is closely related with the unfolding scheme 
used in Ref. 52 and up to some generalizations and approximations it is equivalent to the unfolding scheme proposed in Refs. 53-56, but the detailed comparison is out of the scope of the current paper.

\section{RESULTS FOR VARYING CORE AND SHELL THICKNESSES}

Now we proceed to the detailed analysis of influence of the crystalline Si shell on electronic structure and density of states (DOS) re-distribution with respect to the Ge NC without shell. We have simulated many different systems that can be separated into two major groups: (a) systems with fixed Ge NC core diameter to $2.9 \mathrm{~nm}$ and variable shell of the thickness $0,0.12,0.25,0.54,0.78,0.93$ and $1.06 \mathrm{~nm}$; (b) set of five different Ge NC core sizes of $1.50,1.75,2.25,2.75$ and $3.50 \mathrm{ML}$ (monolayers), which corresponds to $1.7,2.0,2.5,3.1$ and $3.9 \mathrm{~nm}$ core diameter, each studied for a set of Si shell thicknesses of $0,0.12$, 0.54 and $0.80 \mathrm{~nm}$. More details on the second set can be found in the Supplementary Information. We model $\mathrm{SiO}_{2}$ as a soft material, as a result the strain at the $\mathrm{NC}$ surface is relaxed. However, the strain between the Ge core and Si shell (which roughly results in compression of Ge core and stretching of Si shell) is fully included in the simulations and contributes to modifications in the band structure.

First we will study fixed Ge NC core size of $3.1 \mathrm{~nm}$ with the Si shell varied between 0 and $1.30 \mathrm{~nm}$ (see Fig. 4(a)). Figure 4(b) presents the energy-resolved LDOS for hole and electron states in the radial direction,

$$
n(r, E)=\frac{1}{r^{2}} \sum_{i} \sum_{n, \alpha}\left|C_{n \alpha}^{(i)}\right|^{2} e^{-\frac{\left(r-r_{n}\right)^{2}}{a_{r}^{2}}} e^{-\frac{E-E_{i}}{a_{E}^{2}}} .
$$

where the index $n$ enumerates the atoms with the distance to the QD center $r_{n}, i$ enumerates different solutions with the energies $E_{i}$ and two broadening parameters $a_{r}=1 \AA$ and $a_{E}=50 \mathrm{meV}$. An extra factor $1 / r^{2}$ is added to (9) to produce the values analogous to the regular DOS that satisfy the condition $n(E) \sim \int n(r, E) d^{3} \mathbf{r}$. Color indicates high (red) to low (blue) DOS and the vertical gray lines denote edges of the core and the shell, respectively, with the shell area shown by the gray rectangle. As expected, for thicker Si shell, electrons become localized in the shell, however, due to small energy offset between $\mathrm{Si}$ and Ge conduction band minima, the localization degree is relatively weak.

It is instructive to analyze the microscopic structure of the confined states also in the wave-vector space. This can be shown by examining the energy-resolved k-space LDOS (the term "fuzzy band structure" has been used in Ref. 52) which is calculated as:

$$
n(\mathbf{k}, E)=\sum_{i} \sum_{l, \tau, \alpha}\left|C_{l \tau \alpha}^{\mathbf{k}(i)}\right|^{2} e^{-\frac{\left(\mathbf{k}-\mathbf{k}_{l}\right)^{2}}{a_{k}^{2}}} e^{-\frac{\left(E-E_{i}\right)^{2}}{a_{E}^{2}}}
$$

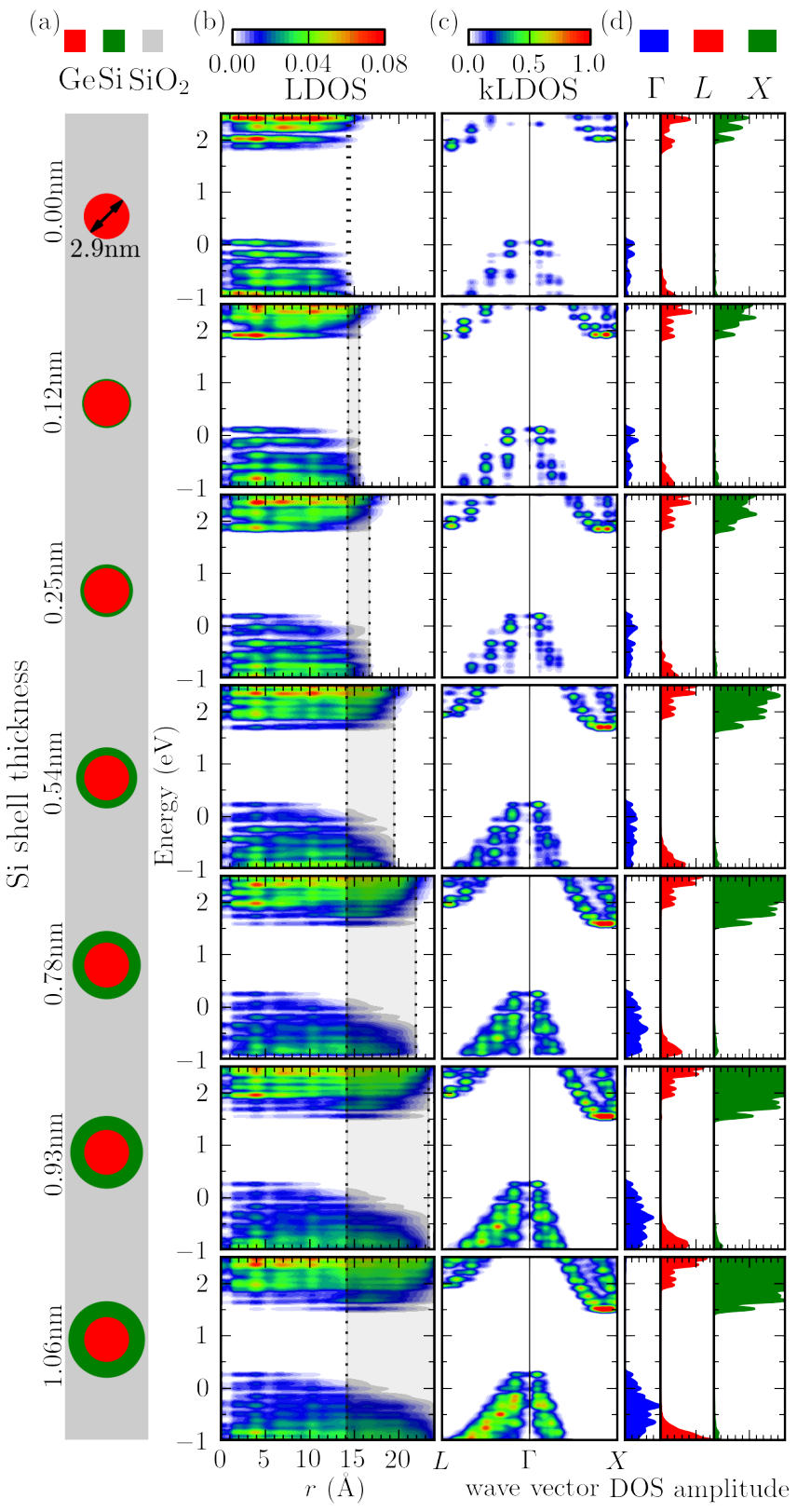

FIG. 4. (Color online) (a) Schematic sketch of the studied $\mathrm{Ge} / \mathrm{Si}$ core/shell NC systems with the fixed core diameter of $2.9 \mathrm{~nm}$ and variable shell thickness between 0.00 and 1.30 nm. (b) real space energy-resolved LDOS, Eq. (9). The grey area shows the position of the Si shell. (c) reciprocal space energy-resolved LDOS, Eq. (10) with $\mathbf{k}$ varying along the $L$ $\Gamma-X$ path. (d) valley-resolved DOS. Three panels show DOS in $\Gamma$ (blue), L (red) or X (green) valley.

where $a_{k}=0.6 \AA^{-1}$ and $a_{E}=50 \mathrm{meV}$. Then we fold $\mathrm{k}$-space onto irreducible part of the Brilllouin zone using its point symmetry and take the 2D $x y$ slice of the function $n(\mathbf{k}, E)$ with $\mathbf{k}$ running along the $L-\Gamma-X$ path in reciprocal space as " $x$ " axis and energy as " $y$ " component. Note that we do not consider integration over angle here, unlike in (9): the real space distribution of LDOS is 

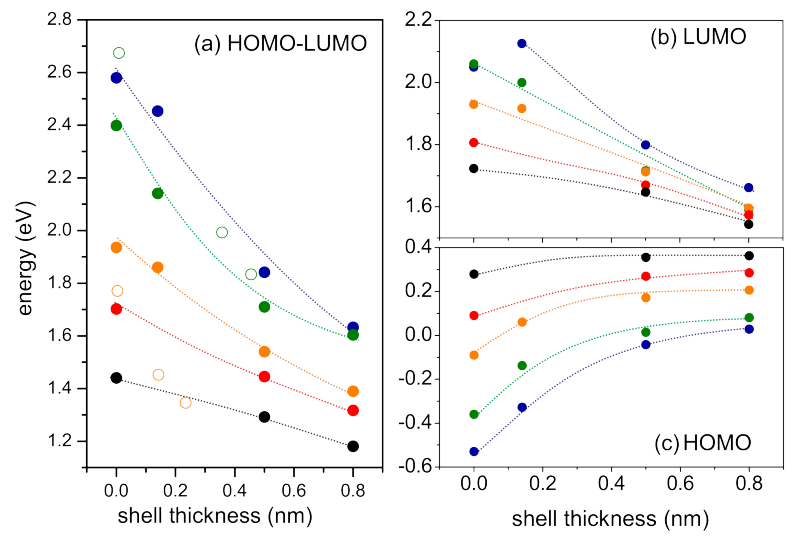

theory: - $2.0 \mathrm{~nm}$ $2.5 \mathrm{~nm}$ - $3.1 \mathrm{~nm}$ - $3.9 \mathrm{~nm}$ experiment: $2.3 \mathrm{~nm}$

FIG. 5. (Color online) (a) Band gap energies and (b) electron (LUMO) and (c) hole (HOMO) energies as a function of the NC core size and shell thickness. Dotted lines serve as the quide to the eye. Empty circle data are experimental data obtained by M. Buljan ${ }^{38}$ for Ge-Si core-shell structures (with not completely enclosed shells).

more or less isotropic while resiprocal space LDOS is noticably localized near valley minima (see Fig. 2(d)). The results of calculation are presented in Fig. 4(c). Colors indicate high (red) to low (blue) DOS. Notably, even in this relatively small Ge NCs, the band structure retains the features of the bulk. When the Si shell thickness increases, the energy of the " $L$-branch" slightly increases and, simultaneously, the " $X$-branch" energy decreases, see Figs. 4(c).

In Fig. 4(d) we evaluate the valley composition of the confined states quantitatively by integrating the density in the reciprocal space in the regions near $X, \Gamma$ and $L$ points. The result is a DOS in three valleys separately. Figure $4(\mathrm{~d})$ shows, that the valence band states are constructed mostly from the states near $\Gamma$ point. On the other hand, the structure of the confined conduction band states changes from $L$-valley states to an $X$-valley states (valley cross-over) when the $\mathrm{Si}$ shell is added and its thickness increases. This effect is more pronounced in smaller Ge NCs and has a threshold behavior.

It is interesting that the $\mathrm{Si}$ shell leads not only to the valley cross-over for the conduction band from $L$ to $X$, but also to a dramatic reduction in the band gap energy of the whole system. Figure 5(a) demonstrates that the NC band gap strongly decreases for larger core diameters as expected from the size quantization effect, but it is also reduced to a similar degree by adding the Si shell only. The shell-induced reduction is more pronounced for smaller NCs. Interestingly, even though holes are confined in the Ge core (Fig. 4(b)), their energy (HOMO) is more sensitive to the shell presence than that for the electronic states (LUMO), which are delocalized over the Ge core and Si shell (more localized in Si for thicker shells), see Fig. 4(b). This is related to larger band offset for the valence band than for conduction band. For thicker Si shell, the spectral tunability by Ge NC core size is reduced when compared to the systems with thinner shell.
We would like to note here, that the native bandgap for uncapped Ge NCs in $\mathrm{SiO}_{2}$ here is reduced with respect to the hydrogen-capped Ge NC in vacuum, as a result of lower potential barrier at the $\mathrm{Si} / \mathrm{SiO}_{2}$ boundary. Optical bandgap energies obtained in our model are compared in Fig. 5(a) with the experimental data obtained in the group of M. Buljan ${ }^{38}$ from the analysis of absorption and structural properties of $\mathrm{Ge} / \mathrm{Si}$ core-shell samples prepared by co-sputtering in $\mathrm{Al}_{2} \mathrm{O}_{3}$ matrix. The calculation in Fig. 5 has been performed for a cubic supercell with the edge length few ML larger than NC diameter (large enough to neglect tunneling through $\mathrm{SiO}_{2}$ ). Theoretical and experimental data appear to agree quantitatively and qualitatively for smaller Ge NCs. For larger NCs the measured band gap is smaller than that predicted here. However, we note that structures prepared by M. Buljan have not fully closed Si shell, as revealed by their in-depth structural analysis by the small-angle X-ray scattering (SAXS). ${ }^{38}$

Now we proceed to the analysis of the radiative recombination rates depending on the thickness of the Si shell for the $3.1 \mathrm{~nm}$ Ge NCs. The phonon-less optical transition rate between the electron states $|e\rangle$ and $|h\rangle$ in the conduction and valence bands is given by ${ }^{57}$

$$
\frac{1}{\tau}=\frac{4 e^{2} \sqrt{\varepsilon_{\text {out }}}\left(E_{e}-E_{h}\right)}{3 \hbar^{2} c^{3}} \mathcal{F}^{2}|\langle e|v| h\rangle|^{2},
$$

where $\mathcal{F}=3 \varepsilon_{\text {out }} /\left(2 \varepsilon_{\text {out }}+\varepsilon_{\text {in }}\right)$ is the local field factor, $E_{e}$ and $E_{h}$ are the energies of the corresponding states. To account for the possible degeneracy of the states the rate Eq. (11) is to be summed over the final states $|h\rangle$ with the same energy and averaged over the initial states $|e\rangle$. The velocity operator $\mathbf{v}$ can be defined as a commutator of the tight-binding Hamiltonian with the coordinate operator, $\mathbf{v}=i[H, \mathbf{r}] / \hbar$. We use the diagonal approximation $\mathbf{r}=\delta_{n n^{\prime}} \delta_{\alpha \beta} \mathbf{r}_{n}$ for the coordinate operator. ${ }^{58-60}$ This corresponds to neglecting the intraatomic matrix elements of the velocity operator for the optical transitions, which is reasonable for covalent semiconductors. ${ }^{61}$

The calculated phonon-less radiative transition rates are presented in Fig. 6. Each transition is shown by a colored dot. To highlight the valley structure of the electron states, the color in Fig. 6(a) indicates the valley attribution of the state, i.e. the blue color corresponds to the electron density over $40 \%$ in the $\Gamma$ point, green to the $X$ point and red to the $L$ point. The figure clearly demonstrates the decrease of the band gap for thicker Si shell, as presented previously in Figs. 4 and 5. Also, the valley cross-over is clearly visible, as the lowest energy transitions change from L-valley (red) to X-valley (green) for thicker shell. Furthermore, addition of shell also leads to dramatic reduction in the rates, which appears to be clearly related to the modification of the $k$-space structure of the electron states.

It is interesting to plot also the real-space-resolved transition rates (Fig. 6(b)) with the color signifying more than $40 \%$ localization of the electron density component in Ge core (red), Si shell (green) or delocalized equally 


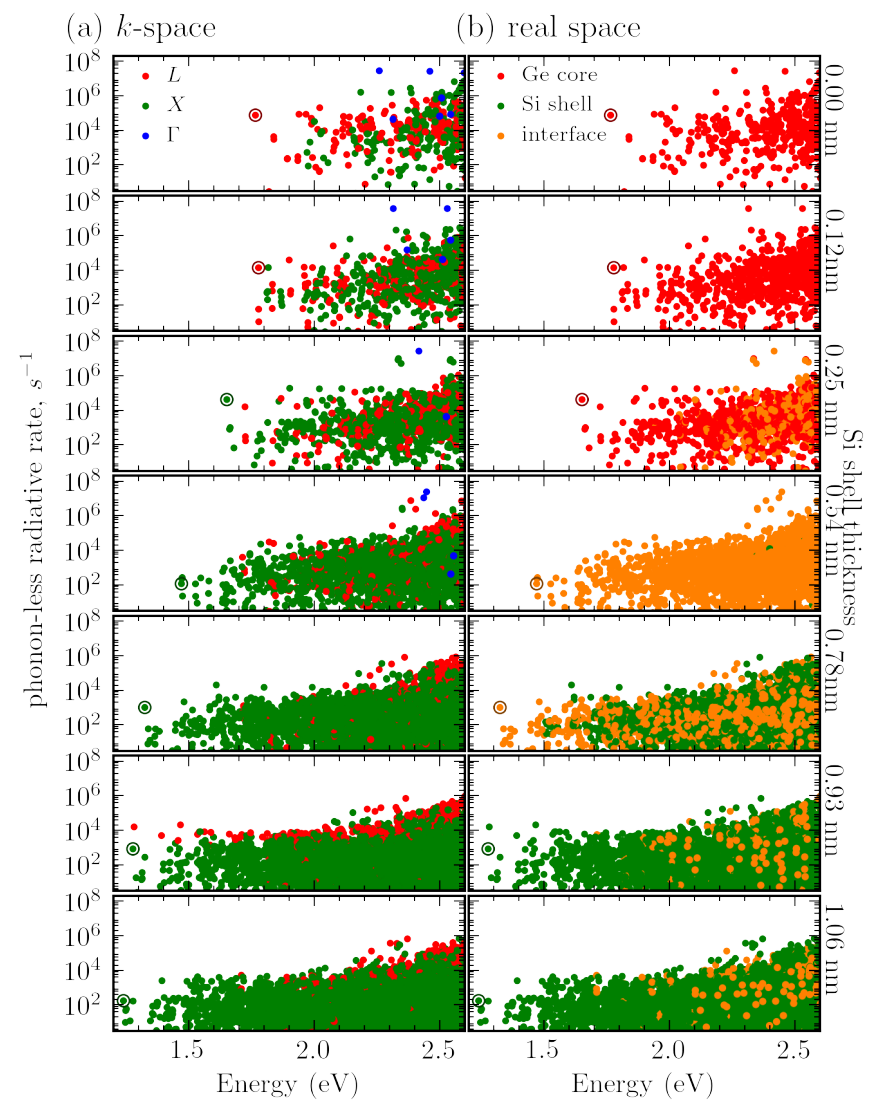

FIG. 6. (Color online) Radiative phonon-less transition rates resolved in color in k-space (a) and real-space (b). Ground state transition is highlighted by circle around it.

in between (orange). This figure shows that even though the localization of electron in the shell in Fig. 4(b) was not clearly pronounced, the rates are already reduced for $0.5 \mathrm{~nm}$-thick Si shell. As such, the rates suppression slightly precedes the over $40 \%$ localization of the electron in the shell, which occurs only for shells thicker than 0.8 $\mathrm{nm}$. This means that the valley cross-over has already a strong influence on the rate reduction beyond the "standard" spatial delocalization role found in typical II-type heterostructures.

\section{CONCLUSIONS}

In our simulation, we present a detailed analysis of $\mathrm{Ge} / \mathrm{Si}$ core-shell NC systems that can be prepared by wet chemical synthesis ${ }^{31}$ or co-sputtering. ${ }^{38}$ New tools have been developed to trace real- and reciprocal-space origin for every transition.

Since the Si shell leads to compressive strain of Ge core, the direct bandgap cannot be induced in such structures. It has been previously shown by the DFT ab-initio simulations ${ }^{40,42}$ that the $\mathrm{Si} / \mathrm{Ge}$ and $\mathrm{Ge} / \mathrm{Si}$ core-shell NC systems are type-II heterostructures with electrons lo- cated in Si and holes in Ge. In our simulations for larger systems, we found that the two phenomena should be distinguished, namely the localization of an electron in the Si shell in the real space and the $L$-to- $X$ valley crossover of the conduction band minimum in the reciprocal space. Localization in the shell is not quite distinct and can be seen only for lowest conduction band states when the shell thickness exceeds approximately $0.7 \mathrm{~nm}$. On the other hand, the valley crossover is already seen for diatomic Si shell. The valence band maximum remains in the $\Gamma$ point for all simulated Si shell thicknesses. Interestingly, even though the holes reside in the Ge core, their energy (HOMO) is influenced by the Si shell more than that of electrons (LUMO). This effect is more pronounced in smaller NCs.

The crossover and associated real space localization of the wavefunction leads to a dramatic reduction in the phonon-less radiative recombination rates. Reduced rates are of interest for photodetector applications. Improved emission yield might be expected for Si-capped NCs, since reduced recombination rate also leads to reduced Auger recombination.

The emission energy of Ge QDs can be efficiently tuned by adding $\mathrm{Si}$ shell of variable thicknesses. For fixed NC core size, strong reduction in band gap energy was observed with thicker Si shell, from visible range towards near-infrared range, interesting for photodetectors and photovoltaics. The optical bandgap energy and shell-dependence are in a good quantitative and qualitative agreement with experimental data obtained by M. Buljan ${ }^{38}$ from samples prepared using co-sputtering technique. For thicker Si shell, the core-size-tunability of the optical bangap is drastically reduced. This is mainly related to strain induced by the Si shell, which modifies the energy offsets of valence and conduction bands differently. This means that the otherwise steep tunability of Ge NCs can be limited by the Si shell, allowing for achieving narrower spectral range for broader-size ensemble of NCs.

In addition to the calculation presented here, the $L$ $X$ crossover may lead to significant change of phononassisted transitions. In order to develop the detailed theory of optical properties of Ge/Se core-shell systems one also needs an accurate consideration of non-radiative recombination at defects in $\mathrm{SiO} 2$ and the renormalization of exciton energy by dielectric confinement in NC. ${ }^{62}$ Both are strongly affected by $\mathrm{Si}$ core thickness. However, it is worth noting that the extremely long radiative decay rates presented in Fig. 6 suggest that in experiments, phonon-assisted recombination bypass phonon-less process.

\section{ACKNOWLEDGMENTS}

The work of MN and AP was financially supported by the Russian Science Foundation grant No. 14-1201067. KD would like to acknowledge financial support by MacGillavry Fellowship at University of Amsterdam. 
1 M. J. Süess, R. Geiger, R. A. Minamisawa, G. Schiefler, J. Frigerio, D. Chrastina, G. Isella, R. Spolenak, J. Faist, and H. Sigg, "Analysis of enhanced light emission from highly strained germanium microbridges," Nature Photonics 7, 466 (2013).

2 Y. M. Niquet, G. Allan, C. Delerue, and M. Lannoo, "Quantum confinement in germanium nanocrystals," Appl. Phys. Lett. 77, 1182-1184 (2000).

${ }^{3}$ C. Bostedt, T. van Buuren, T. M. Willey, N. Franco, L. J. Terminello, C. Heske, and T. Möller, "Strong quantumconfinement effects in the conduction band of germanium nanocrystals," Applied Physics Letters 84, 4056 (2004).

4 C. Bulutay, "Interband, intraband, and excited-state direct photon absorption of silicon and germanium nanocrystals embedded in a wide band-gap lattice," Phys. Rev. B 76, 205321 (2007).

5 C. Sevik and C. Bulutay, Phys. Rev. B 77, 125414 (2008).

6 S. Ossicini, M. Amato, R. Guerra, M. Palummo, and O. Pulci, Nanoscale Res. Lett. 5, 1637 (2010).

7 Y. Maeda, Phys. Rev. B 51, 1658 (1995).

8 S. Takeoka, M. Fujii, S. Hayashi, and K. Yamamoto, "Size-dependent near-infrared photoluminescence from Ge nanocrystals embedded in $\mathrm{SiO}_{2}$ matrices," Phys. Rev. B 58, 7921-7925 (1998).

9 R. Chivas, S. Yerci, R. Li, L. Dal Negro, and T. F. Morse, Opt. Mater. 33, 1829 (2011).

10 S. Saeed, E. M. L. D. de Jong, K. Dohnalova, and T. Gregorkiewicz, "Efficient optical extraction of hot-carrier energy," Nature Communications 5, 4665 (2014).

11 Mayeul d'Avezac, Jun-Wei Luo, Thomas Chanier, and Alex Zunger, "Genetic-algorithm discovery of a direct-gap and optically allowed superstructure from indirect-gap $\mathrm{Si}$ and Ge semiconductors," Phys. Rev. Lett. 108, 027401 (2012).

12 Lijun Zhang, Mayeul dAvezac, Jun-Wei Luo, and Alex Zunger, "Genomic design of strong direct-gap optical transition in $\mathrm{Si} / \mathrm{Ge}$ core/multishell nanowires," Nano letters 12, 984 (2012).

13 S. Kolodinski, J. H. Werner, and H.J. Queisser, Appl. Phys. A 61, 535 (1995).

14 M. Wolf, R. Brendel, J. H. Werner, and H. J. Queisser, J. Appl. Phys. 83, 4213 (1998).

15 H. J. Queisser J. H. Werner, S. Kolodinski, Phys. Rev. Lett. 72, 3851 (1994).

16 Chris G. Van de Walle, "Band lineups and deformation potentials in the model-solid theory," Phys. Rev. B 39, 1871-1883 (1989).

17 Yijie Huo, Hai Lin, Robert Chen, Maria Makarova, Yiwen Rong, Mingyang Li, Theodore I. Kamins, Jelena Vuckovic, and James S. Harris, "Strong enhancement of direct transition photoluminescence with highly tensile-strained ge grown by molecular beam epitaxy," Applied Physics Letters 98, 011111 (2011).

18 Jinendra Raja Jain, Aaron Hryciw, Thomas M. Baer, David A. B. Miller, Mark L. Brongersma, and Roger T. Howe, "A micromachining-based technology for enhancing germanium light emission via tensile strain," Nature Photonics 6, 398 (2012).

19 Hai Liu, Wyatt Winkenwerder, Yueran Liu, Domingo Ferrer, Davood Shahrjerdi, Scott K. Stanley, John G. Ekerdt, and Sanjay K. Banerjee, "Core-shell germanium- silicon nanocrystal floating gate for nonvolatile memory applications," IEEE TRANSACTIONS ON ELECTRON DEVICES 55, 2610 (2008).

${ }^{20}$ K. S. Min, K. V. Shcheglov, C. M. Yang, H. A. Atwater, M. L. Brongersma, and A. Polman, Appl. Phys. Lett. 68, 2511 (1996).

21 X. L. Wu, T. Gao, G. G. Siu, S. Tong, and X. M. Bao, Appl. Phys. Lett. 74, 2420 (1999).

22 S. Takeoka, K. Toshikiyo, M. Fujii, S. Hayashi, and K. Yamamoto, Phys. Rev. B 61, 15988 (2000).

23 Christophe Galland, Yagnaseni Ghosh, Andrea Steinbrück, Jennifer A. Hollingsworth, Han Htoon, and Victor I. Klimov, "Lifetime blinking in nonblinking nanocrystal quantum dots," Nature Communications 3, 908 (2012).

24 Victor I. Klimov, Sergei A. Ivanov, Jagjit Nanda, Marc Achermann, Ilya Bezel, John A. McGuire, and Andrei Piryatinski1, "Single-exciton optical gain in semiconductor nanocrystals," Nature 447, 441 (2007).

25 Saba Saeed, Frank Buters, Katerina Dohnalova, Lech Wosinski, and Tom Gregorkiewicz, "Structural and optical characterization of self-assembled ge nanocrystal layers grown by plasma-enhanced chemical vapor deposition," Nanotechnology 25, 405705 (2014).

${ }^{26}$ X. W. Wang, H. Lee, Y. C. Lan, G. H. Zhu, G. Joshi, D. Z. Wang, J. Yang, A. J. Muto, M. Y. Tang, J. Klatsky, S. Song, M. S. Dresselhaus, G. Chen, and Z. F. Ren, Appl. Phys. Lett. 93, 193121 (2008).

27 A. Malachias, S. Kycia, G. Medeiros-Ribeiro, R. Magalhaes-Paniago, T. I. Kamins, and R. S. Williams, Phys. Rev. Lett. 91, 176101 (2003).

28 M. I. Alonso, M. D. Calle, J. O. Osso, M. Garriga, and A. R. Goni, J. Appl. Phys. 98, 033530 (2005).

29 M. Y. Valakh, V. O. Yukhymchuk, V. M. Dzhagan, O. S. Lytvyn, A. G.Milekhin, O. P. Pchelyakov, F. Alsina, and J. Pascual, Nanotechnology 16, 1464 (2005).

30 I. N. Demchenko, K. Lawniczak-Jablonska, S. Kret, A. V. Novikov, J. Y. Laval, M. Zak, A. Szczepanska, A. N. Yabloskiy, and Z. F. Krasilnik, Nanotechnology 18, 115711 (2007).

31 C.-S. Yang, S. Kauzlarich, and Y. C. Wang, Chem. Mater. 11, 3666 (1999).

32 S. D. Barry, Z. Yang, J. A. Kelly, E. J. Henderson, and J. G. C. Veinot, Chem. Mater. 23, 5096 (2011).

33 X. D. Pi and U. Kortshagen, Nanotechnology 20, 295602 (2009).

34 F. Erogbogbo, T. Liu, N. Ramadurai, P. Tuccarione, L. Lai, M. T. Swihart, and P. N. Prasad, ACS Nano 5, 7950 (2011).

35 O. Yasar-Inceoglu and L. Mangolini, Mater. Lett. 101, 76 (2013).

36 Christian Mehringer, Christian Kloner, Benjamin Butz, Benjamin Winter, Erdmann Spiecker, and Wolfgang Peukert, "Germanium-silicon alloy and core-shell nanocrystals by gas phase synthesis," Nanoscale 7, 5186-5196 (2015).

37 S. Takeoka, K. Toshikiyo, M. Fujii, S. Hayashi, and K. Yamamoto, J. Lumin. 87-89, 352 (2000).

38 M. Buljan, S. R. C. Pinto, A. G. Rolo, J. Martín-Sánchez, M. J. M. Gomes, J. Grenzer, A. Mücklich, S. Bernstorff, and V. Holý, "Self-assembling of Ge quantum dots in an alumina matrix," Phys. Rev. B 82, 235407 (2010).

39 A. Kolobov, H. Oyanagi, N. Usami, S. Tokumitsu, T. Hat- 
tori, S. Yamasaki, K. Tanaka, S. Othake, and Y. Shiraki, Appl. Phys. Lett. 80, 488 (2002).

${ }^{40}$ L. E. Ramos, J. Furthmüller, and F. Bechstedt, "Quantum confinement in Si- and Ge-capped nanocrystallites," Phys. Rev. B 72, 045351 (2005).

${ }^{41}$ Y. M. Yang, X. L. Wu, G. G. Su, G. S. Huang, J. C. Shen, and D. S. Hu, J. Appl. Phys. 96, 5239 (2004).

42 E. L. de Oliveira, E. L. Albuquerque, J. S. de Sousa, G. A. Farias, and F. M. Peeters, "Configuration-Interaction Excitonic Absorption in Small Si/Ge and Ge/Si Core/Shell Nanocrystals," J. Phys. Chem. C 116, 4399-4407 (2012).

${ }^{43}$ M. R. Neupane, R. K. Lake, and R. Rahman, "Core size dependence of the confinement energies, barrier heights, and hole lifetimes in Ge-core/Si-shell nanocrystals," J. Appl. Phys. 110, 074306 (2011).

44 Mahesh R. Neupane, Rajib Rahman, and Roger K. Lake, "Effect of strain on the electronic and optical properties of Ge - Si dome shaped nanocrystals," Phys. Chem. Chem. Phys 17, 2484 (2015).

45 P. N. Keating, "Effect of invariance requirements on the elastic strain energy of crystals with application to the diamond structure," Phys. Rev. 145, 637-645 (1966).

46 H. Rücker and M. Methfessel, "Anharmonic Keating model for group-iv semiconductors with application to the lattice dynamics in alloys of Si, Ge, and C," Phys. Rev. B 52, 11059-11072 (1995).

47 Jean-Marc Jancu, Reinhard Scholz, Fabio Beltram, and Franco Bassani, "Empirical spds* tight-binding calculation for cubic semiconductors: General method and material parameters," Phys. Rev. B 57, 6493-6507 (1998).

48 Y. M. Niquet, D. Rideau, C. Tavernier, H. Jaouen, and $\mathrm{X}$. Blase, "Onsite matrix elements of the tight-binding hamiltonian of a strained crystal: Application to silicon, germanium, and their alloys," Phys. Rev. B 79, 245201 (2009).

49 Yuhai Tu and J. Tersoff, "Structure and Energetics of the Si- sion Interface," Phys. Rev. Lett. 84, 4393-4396 (2000).

50 H. Watanabe, K. Kawabata, and T. Ichikawa, "A Tight Binding Method Study of Optimized System," Electron Devices, IEEE Transactions on 57, 3084 -3091 (2010).

51 G. Bricogne, "International tables for crystallography," (D. Reidel Publishing Company, 2006) Chap. Fourier transforms in crystallography: theory, algorithms and applications.

52 P. Hapala, K. Kůsová, I. Pelant, and P. Jelínek, "Theoretical analysis of electronic band structure of 2- to 3-nm Si nanocrystals," Phys. Rev. B 87, 195420 (2013).

53 B. T. Boykin and G. Klimeck, "Practical application of zone-folding concepts in tight-binding calculations," Phys. Rev. B 71, 115215 (2005).

54 Timothy B Boykin, Neerav Kharche, Gerhard Klimeck, and Marek Korkusinski, "Approximate bandstructures of semiconductor alloys from tight-binding supercell calculations," J. Phys.: Conden. Matt. 19, 036203 (2007).

${ }^{55} \mathrm{~V}$. Popescu and A. Zunger, "Effective band structure of random alloys," Phys. Rev. Lett. 104, 236403 (2010).

56 V. Popescu and A. Zunger, "Extracting $E$ versus $\vec{k}$ effective band structure from supercell calculations on alloys and impurities," Phys. Rev. B 85, 085201 (2012).

57 C. Delerue and M. Lannoo, Nanostructures. Theory and Modelling (Springer Verlag, Berling, Heidelberg, 2004).

${ }^{58}$ L. C. Lew Yan Voon and L. R. Ram-Mohan, "Tightbinding representation of the optical matrix elements:
Theory and applications," Phys. Rev. B 47, 15500-15508 (1993).

59 E.L. Ivchenko and M.O. Nestoklon, "Optical transitions on a type II semiconductor interface in the empirical tightbinding theory," Journal of Experimental and Theoretical Physics 94, 644-653 (2002).

60 T. Sandu, "Optical matrix elements in tight-binding models with overlap," Phys. Rev. B 72, 125105 (2005).

61 M. Cruz, M. R. Beltrán, C. Wang, J. Tagüeña-Martínez, and Yuri G. Rubo, "Supercell approach to the optical properties of porous silicon," Phys. Rev. B 59, 15381-15387 (1999).

${ }^{62}$ R. Benchamekh, N. A. Gippius, J. Even, M. O. Nestoklon, J.-M. Jancu, S. Ithurria, B. Dubertret, Al. L. Efros, and P. Voisin, "Tight-binding calculations of image-charge effects in colloidal nanoscale platelets of cdse," Phys. Rev. B 89, 035307 (2014).

${ }^{63}$ F Raouafi, R Benchamekh, M O Nestoklon, J-M Jancu, and $\mathrm{P}$ Voisin, "Intrinsic interface states in inasalsb heterostructures," Journal of Physics: Condensed Matter 28, 045001 (2016).

64 M.O. Nestoklon, R. Benchamekh, and P. Voisin, "Virtual crystal description of III-V semiconductor alloys in the tight binding approach," Journal of Physics: Condensed Matter ??, ??? (2016). 


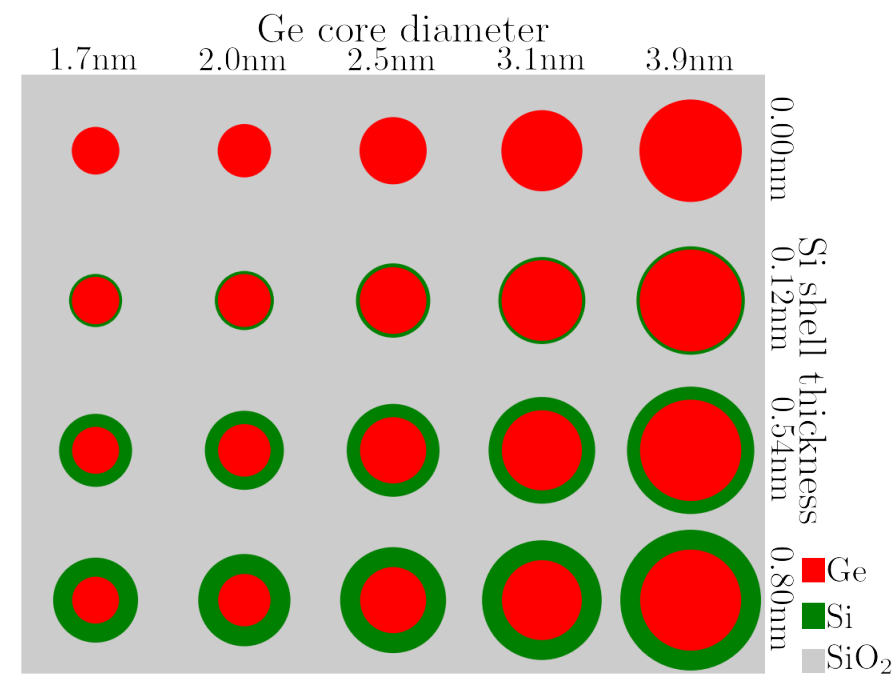

FIG. 7. (Color online) Schematic sketch of the simulated structures of $\mathrm{Ge} / \mathrm{Si}$ core/shell NCs systems: a set of $5 \mathrm{Ge}$ $\mathrm{NC}$ core sizes of 1.7, 2.0, 2.5, 3.1 and $3.9 \mathrm{~nm}$, each core size including Si shell thicknesses 0.00, 0.14, 0.50 and $0.80 \mathrm{~nm}$.

\section{SUPPLEMENTARY}

In supplementary material we present calculations for the core-shell NCs for 5 Ge core sizes of 1.7, 2.0, 2.5, 3.1 and $3.9 \mathrm{~nm}$ and for the Si shell thicknesses $0.00,0.14$, 0.50 and $0.80 \mathrm{~nm}$. The systems are sketched in Fig. 7.

Figure 8 shows the real-space projection of the electronic density for hole and electron states. Color indicates high (red) to low (blue) DOS and the vertical gray lines denote edge of the core and shell, respectively, with the shell area in gray rectangle.

Figure 9 shows the reciprocal space projection of the electronic density for hole and electron states. Color indicates high (red) to low (blue) DOS.

In Fig. 10 we show the quantitative valley compositions obtained by integrating the reciprocals-space carrier density in the regions near $\Gamma, X$ and $L$ points. To show the whole range of the DOS in all plots, we multiply the calculated DOS to the factor which is indicated in the right bottom corner of each panel.

The calculated phonon-less radiative transition rates are presented in Fig. 11. Each transition is shown by a colored dot. In order to visualize the valley structure of the electron states, the color shows the amplitude (over $40 \%$ component) in the corresponding valleys, i.e. the blue component corresponds to the electron density from over $40 \%$ in $\Gamma$ point, green component to $X$ point and red to $L$ point.

In Fig. 12 we show the real-space-colored transition rates with color signifying more than $40 \%$ localization of the electron density component in Ge core (red), Si shell (green) or delocalized equally in between (orange). 


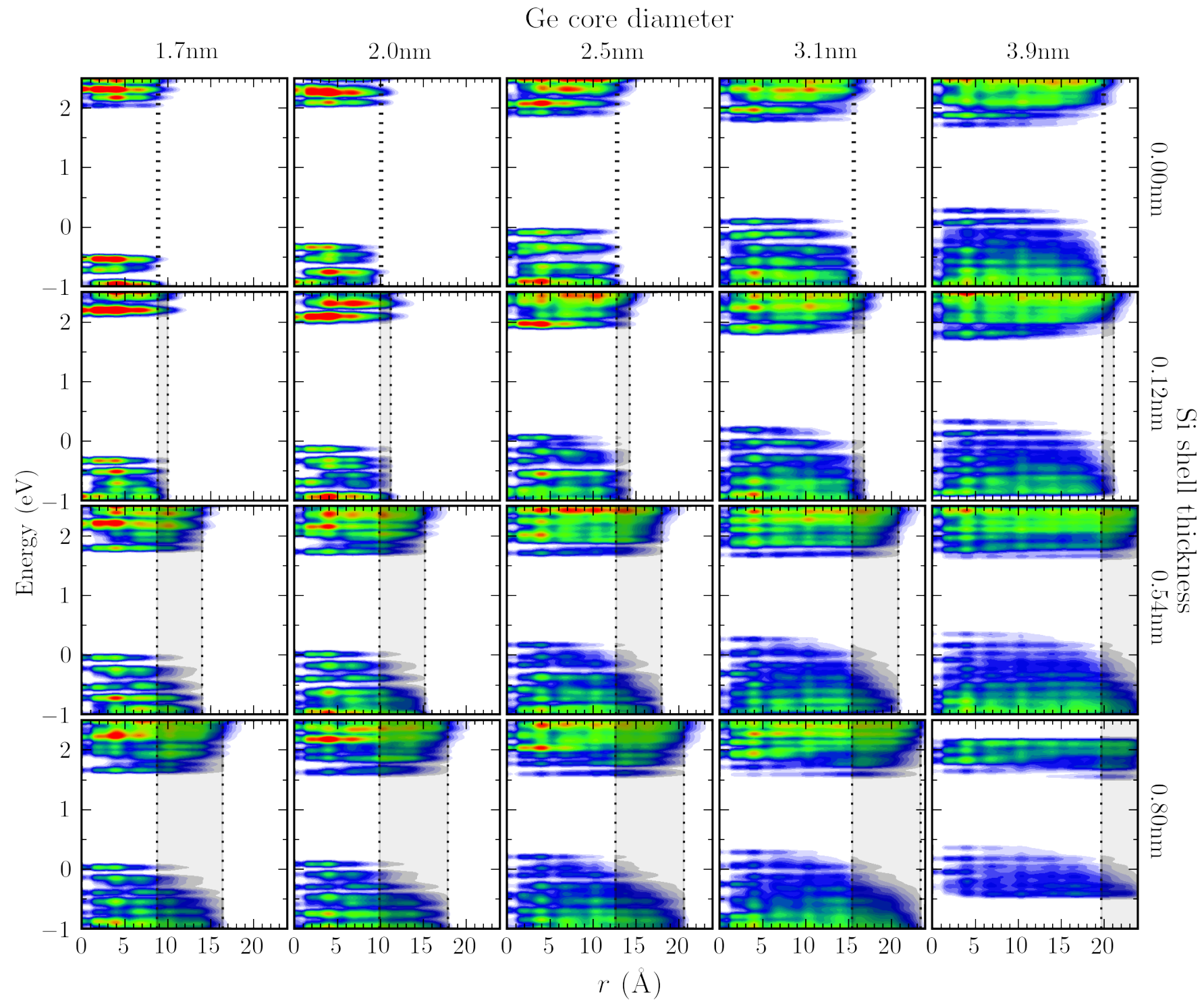

FIG. 8. (Color online) Real-space resolved density of states for electrons and holes. Similar to Fig. 4(b), but for the set of NCs with changing core radius and shell thickness 


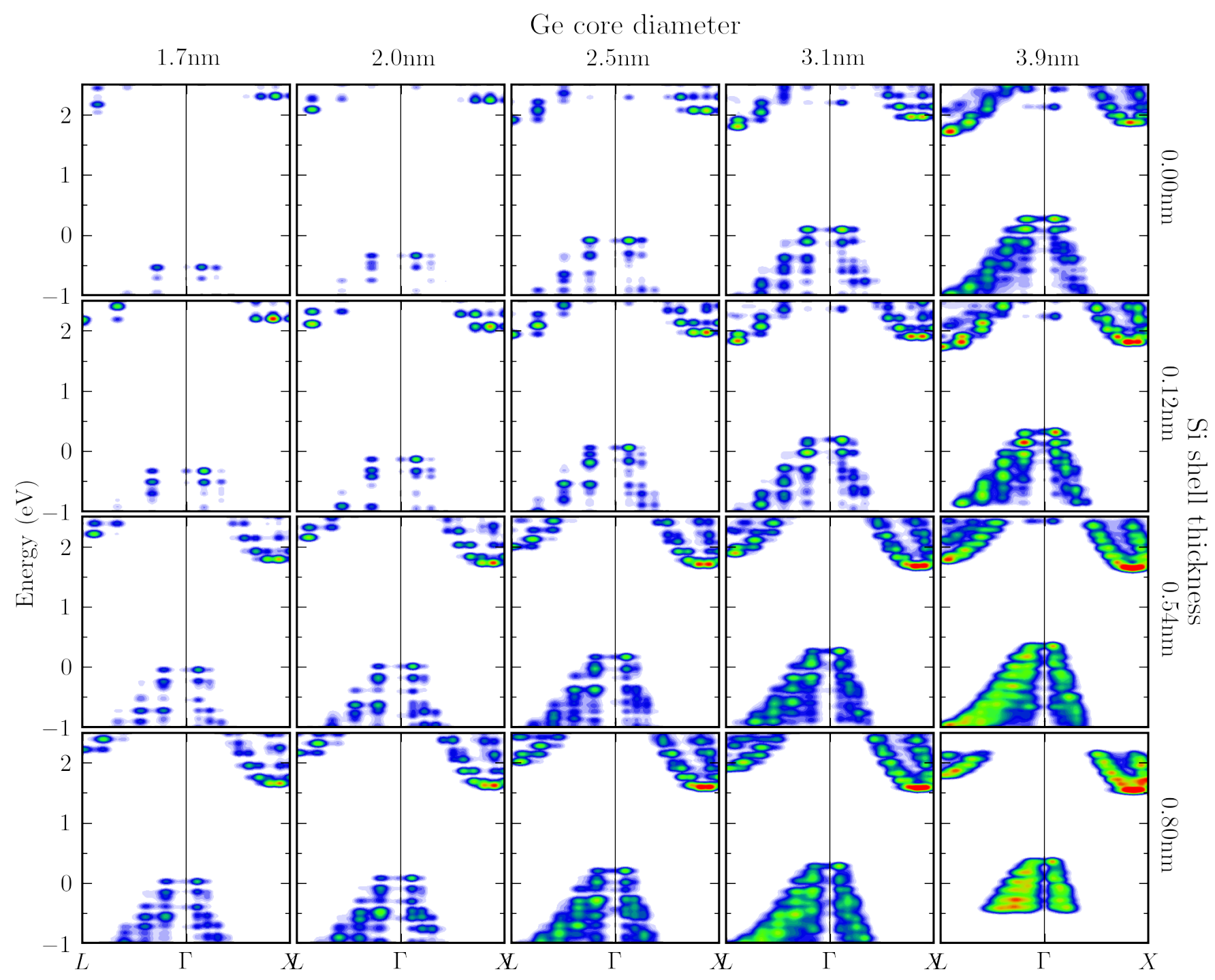

FIG. 9. (Color online) $k$-space resolved density of states for electrons and holes. Similar to Fig. 4 (c), but for the set of NCs with changing core radius and shell thickness 
Ge core diameter
$1.7 \mathrm{~nm}$
$2.0 \mathrm{~nm}$
$2.5 \mathrm{~nm}$
$3.1 \mathrm{~nm}$
$3.9 \mathrm{~nm}$

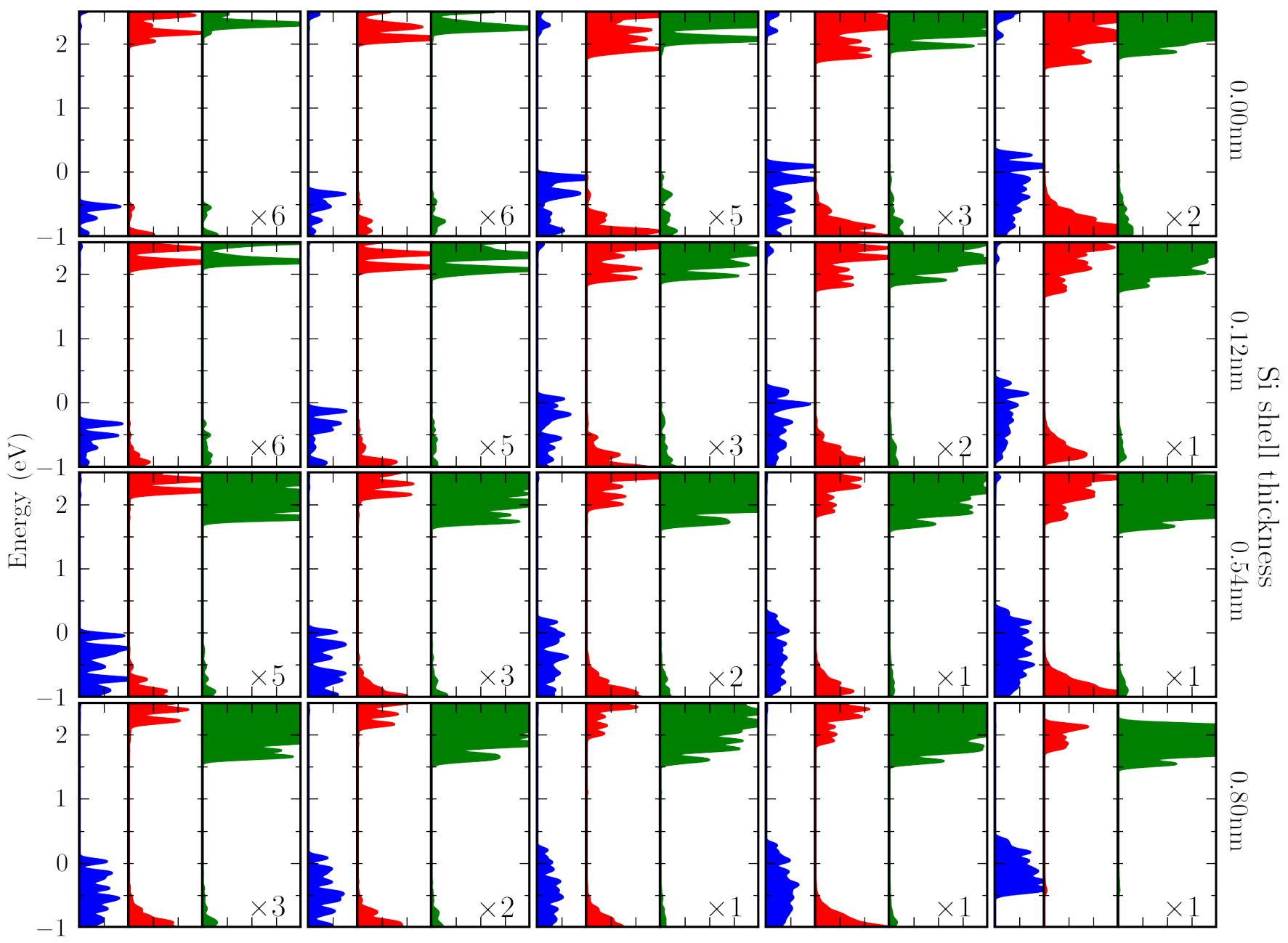

FIG. 10. (Color online) Valley-resolved DOS as a function of both Ge core diameter and Si shell thickness. Similar to Fig. 4(d), but for the set of NCs with changing core radius and shell thickness. To show the whole range of the DOS in all plots, we multiply the calculated DOS to the factor which is indicated in the right bottom corner of each panel. 
Ge core diameter

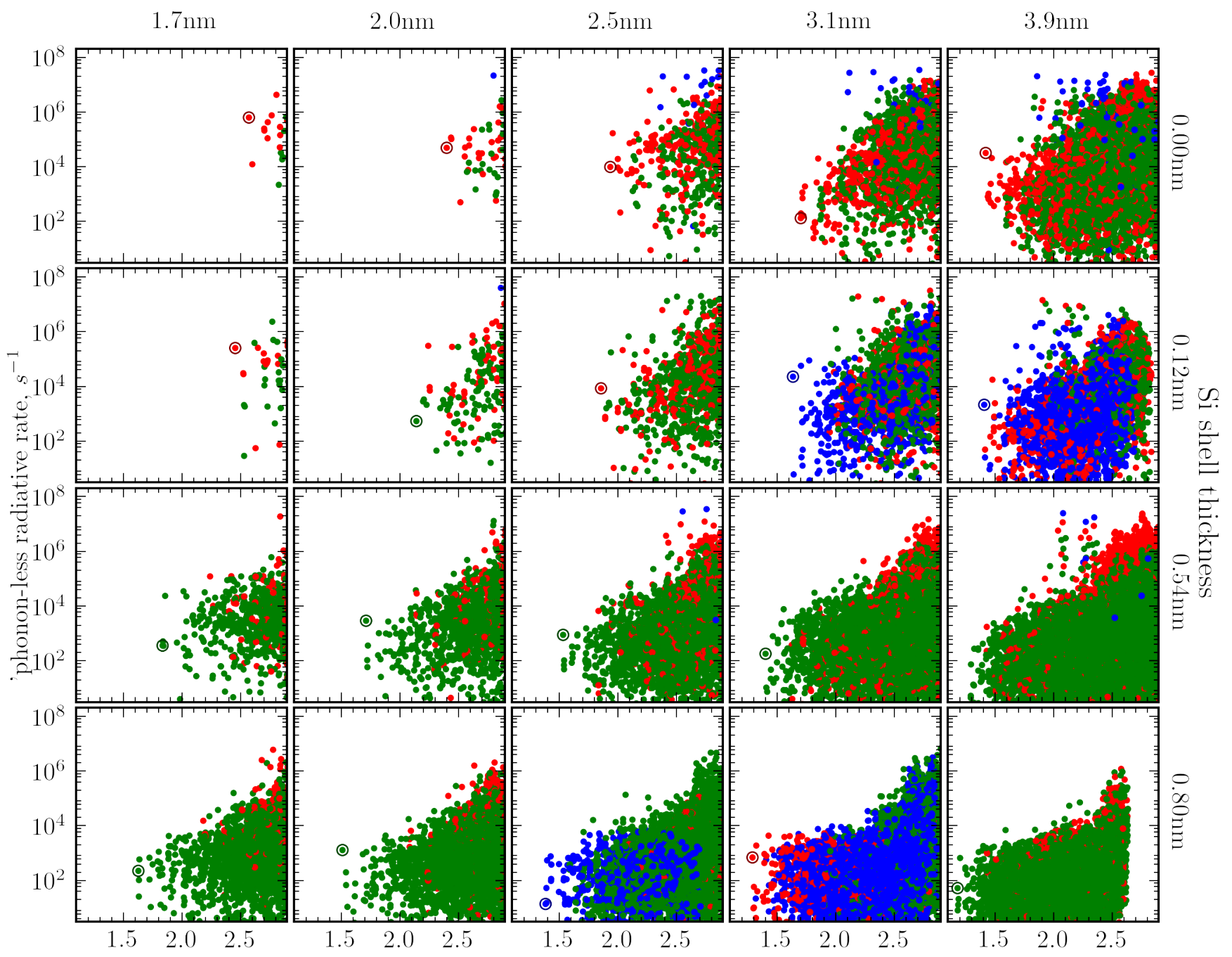

FIG. 11. (Color online) Radiative phonon-less transition rates resolved in k-space. Similar to the Fig. 6(a). 
Ge core diameter

$1.7 \mathrm{~nm}$

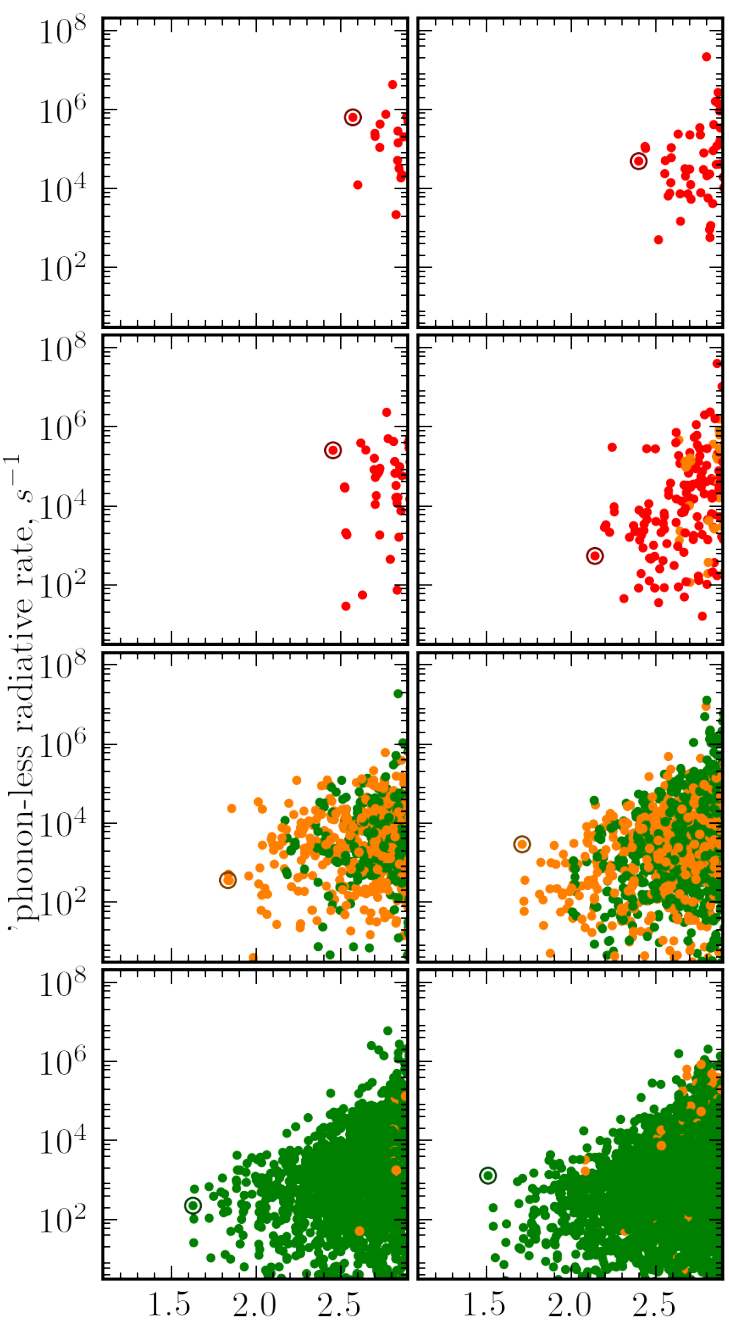

$2.5 \mathrm{~nm}$
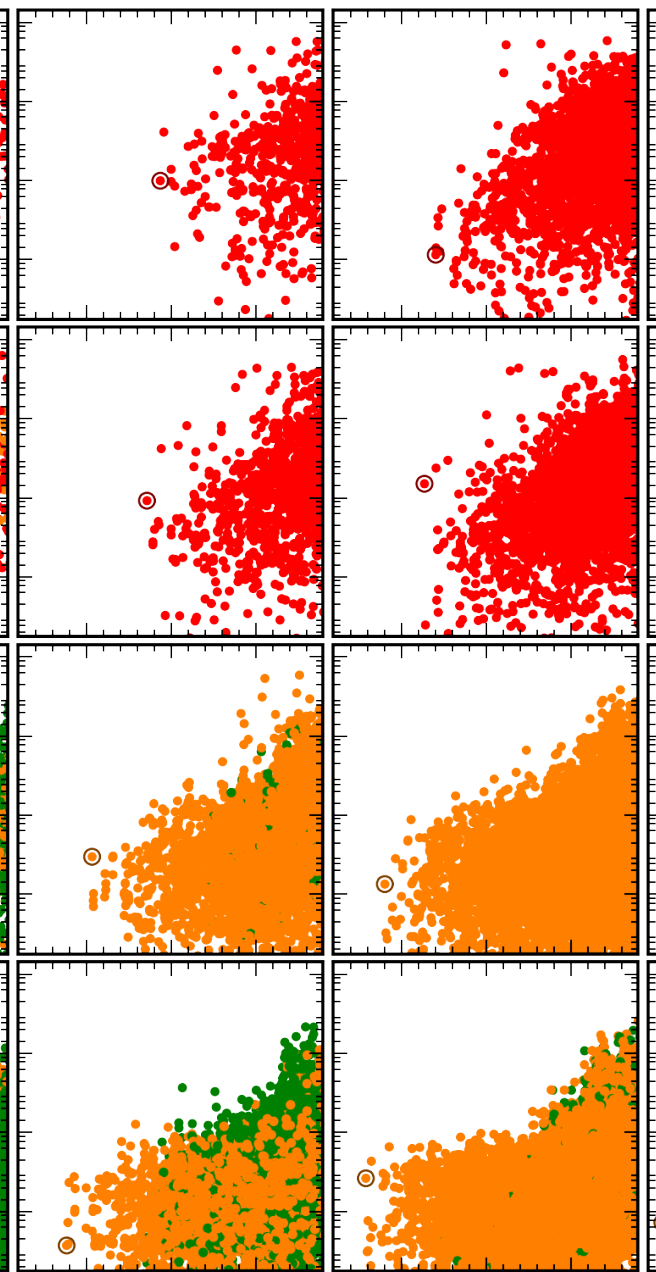

$\begin{array}{lll}1.5 & 2.0 & 2.5\end{array}$
$3.1 \mathrm{~nm}$

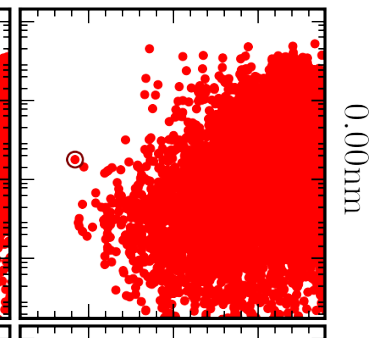

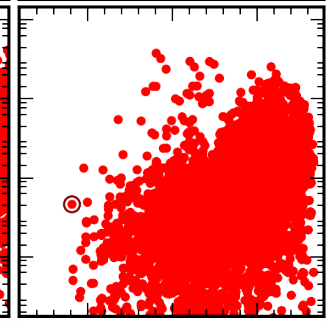

总

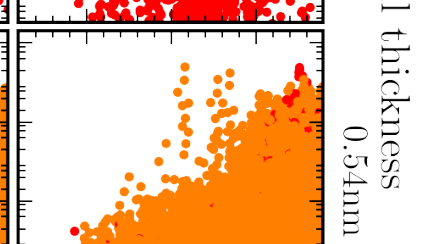

FIG. 12. (Color online) Radiative phonon-less transition rates resolved in color in real-space. Similar to the Fig. 6(b). 
TABLE I. Tight-binding parameters of $\mathrm{Si}, \mathrm{Ge}$ and " $\mathrm{SiO}_{2}$ " used in calculations. Si and Ge parameters are taken from Ref. 48 and $\mathrm{SiO}_{2}$ are fit to reproduce bandstructure of $\alpha$ quartz.

\begin{tabular}{lrrr}
\hline & $\mathrm{Si}$ & $\mathrm{Ge}$ & \multicolumn{1}{c}{$\mathrm{SiO}_{2} "$} \\
\hline$a$ & 5.4300 & 5.6500 & \\
$E_{s}$ & -2.5525 & -3.4025 & 1.3859 \\
$E_{s^{*}}$ & 23.4461 & 23.8817 & 27.4887 \\
$E_{p}$ & 4.4859 & 5.3147 & 1.3391 \\
$E_{d}$ & 14.0105 & 12.8753 & 15.1568 \\
\hline$s s \sigma$ & -1.8660 & -1.4909 & -2.7825 \\
$s^{*} s^{*} \sigma$ & -4.5133 & -4.8612 & -5.6494 \\
$s s^{*} \sigma$ & -1.3911 & -1.5948 & -1.7632 \\
$s p \sigma$ & 2.9107 & 2.9128 & 3.4027 \\
$s^{*} p \sigma$ & 3.0682 & 2.9204 & 3.1798 \\
$s d \sigma$ & -2.2399 & -2.1011 & -4.6536 \\
$s^{*} d \sigma$ & -0.7771 & -0.2356 & -1.5761 \\
$p p \sigma$ & 4.0848 & 4.3662 & 5.1413 \\
$p p \pi$ & -1.4921 & -1.5831 & -0.5472 \\
$p d \sigma$ & -1.6666 & -1.6011 & -2.3762 \\
$p d \pi$ & 2.3994 & 2.3698 & 1.2601 \\
$d d \sigma$ & -1.8295 & -1.1548 & -2.7520 \\
$d d \pi$ & 3.0818 & 2.3004 & 1.1614 \\
$d d \delta$ & -1.5668 & -1.1939 & -0.6707 \\
\hline$\Delta / 3$ & 0.0185 & 0.1274 & 0.0000
\end{tabular}

TABLE II. Tight-binding of SiGe used in calculations. Parameters are taken from Ref. 48. For SiGe diagonal energies and spin-orbit constants are the same as for $\mathrm{Si}$ and $\mathrm{Ge}$, we present only transfer matrix elements here.

\begin{tabular}{lrrr}
\hline \multicolumn{3}{c}{$\operatorname{Si}(1) \mathrm{Ge}(2)$} & \\
\hline$s s \sigma$ & -1.6765 & & \\
$s^{*} s^{*} \sigma$ & -4.6335 & & \\
$p p \sigma$ & 4.2193 & & \\
$p p \pi$ & -1.5467 & & \\
$d d \sigma$ & -1.4195 & & \\
$d d \pi$ & 2.6254 & & -1.5031 \\
$d d \delta$ & -1.3938 & & 2.8289 \\
$s_{1} s_{2}^{*} \sigma$ & -1.5094 & $s_{2} s_{1}^{*} \sigma$ & -2.0630 \\
$s_{2} p_{1} \sigma$ & 3.0103 & $s_{1} p_{2} \sigma$ & -0.4639 \\
$s_{2}^{*} p_{1} \sigma$ & 2.7930 & $s_{1}^{*} p_{2} \sigma$ & -1.4341 \\
$s_{2} d_{1} \sigma$ & -2.0474 & $s_{2} d_{1} \sigma$ & 2.5711 \\
$s_{2}^{*} d_{1} \sigma$ & -0.5123 & $s_{2}^{*} d_{1} \sigma$ & \\
$p_{2} d_{1} \sigma$ & -1.6132 & $p_{1} d_{2} \sigma$ & $p_{2} d_{1} \pi$ \\
$p_{2} d_{1} \pi$ & 2.4355 & &
\end{tabular}

\section{TIGHT-BINDING PARAMETERS}

In Table I we present tight-binding parameters used in calculations.

For the strain we use parameters from Table III. We

TABLE III. Tight-binding strain parameters used in calculations. Si, Ge and SiGe parameters are taken from ${ }^{48}$, but we used simplified version of strain treatment and parameters $\beta$ replaced with $\lambda$ which have similar meaning. Tight-binding parameters of " $\mathrm{SiO}_{2}$ " are not renormalized by the strain.

\begin{tabular}{|c|c|c|c|}
\hline & $\mathrm{Si}$ & $\mathrm{Ge}$ & $\mathrm{SiGe}$ \\
\hline$n_{s s \sigma}$ & 3.5670 & 3.5744 & 3.9017 \\
\hline$n_{s s * \sigma}$ & 1.5197 & 1.0363 & 1.0380 \\
\hline$n_{s p \sigma}$ & 2.0353 & 2.8820 & 2.3728 \\
\hline$n_{s d \sigma}$ & 2.1481 & 1.8928 & 1.9954 \\
\hline$n_{s * s * \sigma}$ & 0.6440 & 1.0794 & 0.8599 \\
\hline$n_{s * p \sigma}$ & 1.4665 & 2.6481 & 1.9414 \\
\hline$n_{s * d \sigma}$ & 1.7967 & 2.3342 & 2.0105 \\
\hline$n_{p p \sigma}$ & 2.0191 & 2.4058 & 2.3500 \\
\hline$n_{p p \pi}$ & 2.8728 & 2.9503 & 3.0815 \\
\hline$n_{p d \sigma}$ & 1.0045 & 0.5132 & 0.7555 \\
\hline$n_{p d \pi}$ & 1.7803 & 1.6242 & 1.6703 \\
\hline$n_{d d \sigma}$ & 1.7387 & 1.6841 & 1.6698 \\
\hline$n_{d d \pi}$ & 1.8044 & 2.6495 & 2.2497 \\
\hline$\underline{n_{d d \delta}}$ & 2.5469 & 3.8322 & 3.0631 \\
\hline$\alpha_{s}$ & -0.1336 & -0.3325 & \\
\hline$\alpha_{p}$ & -0.1895 & -0.4382 & \\
\hline$\alpha_{d}$ & -0.8905 & -0.9049 & \\
\hline$\alpha_{s *}$ & -0.2437 & -0.5206 & \\
\hline$\lambda_{1 p}$ & 0.1389 & -0.3103 & \\
\hline$\lambda_{2 p}$ & 0.0000 & 0.4800 & \\
\hline$\lambda_{1 d}$ & 0.4394 & 0.2434 & \\
\hline
\end{tabular}

use simplified version of approach from Ref. 48, instead parameters $\beta$ we use parameters $\lambda$ explained in Refs. ${ }^{63,64}$ 